\title{
ORT. 16 - Influence of differents cryoprotecttive agents on the maintenance of Vero cells adapted to serum-free culture media
}

Kelly Araujo Lucio ${ }^{1 *}$; Viviane Silva Gomes Rodrigues ${ }^{1}$; Guilherme de Jesus da Silva ${ }^{1}$; Mariana Pierre de Barros Gomes ${ }^{1}$; Marta Cristina de Oliveira Souza ${ }^{1}$; Sheila Maria Barbosa de Lima ${ }^{1}$. 1Fiocruz/Bio-Manguinhos.

Introduction: Cryoprotective agents (CPAs) play a central role in allowing cells to be stored at deep cryogenic temperatures. Most used protocols for cryopreservation of animal cells employ DMSO or Glicerol as CPAs, which have toxic effects for the cells, in medium supplemented with FBS for nutrient supply and stress shielding effects. The removal of animal-derived inputs from the manufacture of immunobiological products is recommended by regulatory agencies around the world. In LATEV, frozen Vero cell banks adapted for growth in serum free medium, contain only culture medium and DMSO, without addition of FBS and it has been observed that cells adapted to serum-free medium to present lag phase after thawing when compared with cells frozen in the presence of DMSO and FBS. The absence of a substance to protect cells may justify such slow growth. The literature has showed that Pluronic F68 (F68) at concentrations of 0.1 to $1 \%$ significantly increased the number of viable cells after thawing.

Objective: The purpose of this work was to evaluate CPAs in the maintenance of Vero cells adapted to culture in serum-free.

Methodology: Vero cells adapted FBS free medium were frozen in the presence of the different CPAs combinations: i) VP-SFM + 10\% DMSO; ii) VP-SFM + 10\% DMSO + 0,1\% F68; iii) $\mathrm{VP}-\mathrm{SFM}+10 \% \mathrm{DMSO}+1 \%$ F 68 ; iv) VP-SFM + 10\% DMSO + 5\% F68; v) VP-SFM + 5\% Gl; vi) VP-SFM + 5\% Gl + 1\% F68; vii) VP-SFM + 5\% Gl + 10\% DMSO; viii) VP-SFM + 5\% Gl $+10 \%$ DMSO $+1 \%$ F68. After thawing, recovery and cell growth in VP-SFM medium ware evaluated for 4 passages.

Results: We evaluated isolated and synergic effect of three CPAs after the thawing Vero cells. The best result obtained were VP-SFM medium in the presence of $10 \%$ DMSO and $10 \%$ DMSO + 1\% F68. Post-thaw viability and morphology were preserved in both situations. At $10 \% \mathrm{DMSO}+5 \% \mathrm{~F} 68$ the protective effect was lost due to the high concentration of F68. In all conditions in which glycerol was added no growth promotion was observed, demonstrating that glycerol is not a good option for Vero cell cryopreservation.

Conclusion: The criopreservation is a valuable tool for cells preservation and the success of this procedure depends on the proper use of CPAs. Although there is no ideal CPA, able to completely protect cells at low temperatures and be free of toxicity, it is clear that only the combination of DMSO and F68 are satisfactory for cryopreserving Vero cells in the absence of FBS and that glycerol is not an option in these tested conditions.

Keywords: cryopreservation; cryoprotective agent; serum-free 